\title{
Lower and upper bounds for strong approximation errors for numerical approximations of stochastic heat equations
}

\author{
Sebastian Becker ${ }^{1}$, Benjamin Gess ${ }^{2,3}$, Arnulf Jentzen ${ }^{4,5}$, \\ and Peter E. Kloeden ${ }^{6}$ \\ ${ }^{1}$ RiskLab, Department of Mathematics, ETH Zurich, \\ 8092 Zurich, Switzerland, e-mail: sebastian.becker@math.ethz.ch \\ 2 Max Planck Institute for Mathematics in the Sciences, \\ 04103 Leipzig, Germany, e-mail: bgess@mis.mpg.de \\ ${ }^{3}$ Faculty of Mathematics, University of Bielefeld, \\ 33615 Bielefeld, Germany, e-mail: benjamin.gess@gmail.com \\ ${ }^{4}$ Faculty of Mathematics and Computer Science, University of Münster, \\ 48149 Münster, Germany, e-mail: ajentzen@uni-muenster.de \\ ${ }^{5}$ SAM, Department of Mathematics, ETH Zurich, \\ 8092 Zurich, Switzerland, e-mail: arnulf.jentzen@sam.math.ethz.ch \\ ${ }^{6}$ Mathematics Institute, Goethe University Frankfurt, \\ 60325 Frankfurt am Main, Germany, e-mail: kloeden@math.uni-frankfurt.de
}

June 17, 2020

\begin{abstract}
This article establishes optimal upper and lower error estimates for strong fulldiscrete numerical approximations of the stochastic heat equation driven by spacetime white noise. Thereby, this work proves the optimality of the strong convergence rates for certain full-discrete approximations of stochastic Allen-Cahn equations with space-time white noise which have been obtained in a recent previous work of the authors of this article.
\end{abstract}




\section{Introduction}

In this work we consider space-time discrete numerical methods for linear stochastic heat partial differential equations of the type

$$
d X_{t}(x)=\Delta X_{t}(x) d t+d W_{t}(x)
$$

with zero Dirichlet boundary conditions $X_{t}(0)=X_{t}(1)=0$ for $t \in[0, T], x \in(0,1)$ where $T \in(0, \infty)$ is the time horizon under consideration and where $\frac{d W}{d t}$ is a spacetime white noise on a probability space $(\Omega, \mathcal{F}, \mathbb{P})$. In particular, we analyse strong rates of convergence of a full-discrete exponential Euler method, proving optimal upper and lower estimates on the strong rate of convergence. The next result, Theorem 1.1 below, summarizes the main findings of this article.

Theorem 1.1. Let $T \in(0, \infty), p \in[2, \infty),\left(H,\langle\cdot, \cdot\rangle_{H},\|\cdot\|_{H}\right)=\left(L^{2}((0,1) ; \mathbb{R}),\langle\cdot, \cdot\rangle_{L^{2}((0,1) ; \mathbb{R})}\right.$, $\left.\|\cdot\|_{L^{2}((0,1) ; \mathbb{R})}\right),\left(P_{n}\right)_{n \in \mathbb{N}} \subseteq L(H)$, let $\left(e_{n}\right)_{n \in \mathbb{N}} \subseteq H$ be an orthonormal basis of $H$, let $A: D(A) \subseteq H \rightarrow H$ be the Laplacian with zero Dirichlet boundary conditions on $H$, assume for all $n \in \mathbb{N}, u \in H$ that $A e_{n}=-\pi^{2} n^{2} e_{n}$ and $P_{n}(u)=\sum_{k=1}^{n}\left\langle e_{k}, u\right\rangle_{H} e_{k}$, let $(\Omega, \mathcal{F}, \mathbb{P})$ be a probability space, let $\left(W_{t}\right)_{t \in[0, T]}$ be an $\operatorname{Id}_{H}$-cylindrical Wiener process, and let $X:[0, T] \times \Omega \rightarrow H$ and $\mathcal{X}^{M, N}:[0, T] \times \Omega \rightarrow H, M, N \in \mathbb{N}$, be stochastic processes which satisfy that for all $t \in[0, T], M \in \mathbb{N}, N \in \mathbb{N}$ it holds $\mathbb{P}$-a.s. that $X_{t}=\int_{0}^{t} e^{(t-s) A} d W_{s}$ and $\mathcal{X}_{t}^{M, N}=\int_{0}^{t} P_{N} e^{(t-\max (\{0, T / M, 2 T / M, \ldots\} \cap[0, s])) A} d W_{s}$. Then there exist $c, C \in(0, \infty)$ such that

(i) we have for all $M \in \mathbb{N}$ that

$$
c M^{-1 / 4} \leq \lim _{n \rightarrow \infty} \sup _{t \in[0, T]}\left(\mathbb{E}\left[\left\|X_{t}-\mathcal{X}_{t}^{M, n}\right\|_{H}^{p}\right]\right)^{1 / p} \leq C M^{-1 / 4}
$$

and

(ii) we have for all $N \in \mathbb{N}$ that

$$
c N^{-1 / 2} \leq \lim _{m \rightarrow \infty} \sup _{t \in[0, T]}\left(\mathbb{E}\left[\left\|X_{t}-\mathcal{X}_{t}^{m, N}\right\|_{H}^{p}\right]\right)^{1 / p} \leq C N^{-1 / 2} .
$$

Theorem 1.1 above is an immediate consequence of Corollary 2.4 below, Lemma 2.6 below, and Da Prato \& Zabczyk [6, Lemma 7.7]. The recent article [1] establishes strong convergence rates for suitable space-time discrete approximation methods for stochastic Allen-Cahn equations of the type

$$
d X_{t}(x)=\Delta X_{t}(x) d t+\left[a X_{t}(x)-b\left(X_{t}(x)\right)^{3}\right] d t+d W_{t}(x)
$$


with zero Dirichlet boundary conditions $X_{t}(0)=X_{t}(1)=0$ for $t \in[0, T], x \in(0,1)$ where $a, b \in[0, \infty)$ are real numbers. Roughly speaking, in [1, Theorem 1.1] a spatial convergence rate of the order $1 / 2-\varepsilon$ and a temporal convergence rate of the order $1 / 4-\varepsilon$ have been established. More precisely, [1, Theorem 1.1] shows that for every $p, \varepsilon \in(0, \infty)$ there exists $C \in \mathbb{R}$ such that for all $M, N \in \mathbb{N}$ we have

$$
\sup _{t \in[0, T]}\left(\mathbb{E}\left[\left\|X_{t}-\mathcal{X}_{t}^{M, N}\right\|_{L^{2}((0,1) ; \mathbb{R})}^{p}\right]\right)^{1 / p} \leq C\left(M^{\left(\varepsilon-\frac{1}{4}\right)}+N^{\left(\varepsilon-\frac{1}{2}\right)}\right)
$$

where $\left(\mathcal{X}_{t}^{M, N}\right)_{t \in[0, T]}$ denotes the nonlinearity-truncated approximation scheme in [1] applied to (4). The results of this article, that is, inequalities (2) and (3), prove that these rates are essentially (up to an arbitrarily small polynomial order of convergence) optimal. We also refer, e.g., to [9, 25, 10, 8, 23, 20, 21, 7, 13, 14, 11, 22, 22, 15, 4, 3, 24, 19] for further research articles on explicit approximation schemes for stochastic differential equations with superlinearly growing non-linearities. Furthermore, related lower bounds for approximation errors in the linear case (i.e., in the case $a=b=0$ in (4)) can, e.g., be found in Müller-Gronbach, Ritter, \& Wagner [17, Theorem 1], Müller-Gronbach \& Ritter [16, Theorem 1], Müller-Gronbach, Ritter, \& Wagner [18, Theorem 4.2], Conus, Jentzen, \& Kurniawan [5, Lemma 6.2], and Jentzen \& Kurniawan [12, Corollary 9.4].

\section{$1.1 \quad$ Acknowledgments}

This work has been partially supported through the SNSF-Research project 200021_156603 "Numerical approximations of nonlinear stochastic ordinary and partial differential equations". The third author acknowledges funding by the Deutsche Forschungsgemeinschaft (DFG, German Research Foundation) under Germanys Excellence Strategy EXC 2044390685587, Mathematics Muenster: Dynamics-Geometry-Structure. B. Gess acknowledges financial support by the DFG through the CRC 1283 "Taming uncertainty and profiting from randomness and low regularity in analysis, stochastics and their applications". 


\section{Lower and upper bounds for strong approxima- tion errors of numerical approximations of linear stochastic heat equations}

\section{$2.1 \quad$ Setting}

Let $\lfloor\cdot\rfloor_{h}: \mathbb{R} \rightarrow \mathbb{R}, h \in(0, \infty)$, be the functions which satisfy for all $h \in(0, \infty), t \in \mathbb{R}$ that $\lfloor t\rfloor_{h}=\max (\{0, h,-h, 2 h,-2 h, \ldots\} \cap(-\infty, t])$. For every measure space $(\Omega, \mathcal{F}, \chi)$, every measurable space $(S, \mathcal{S})$, every set $R$, and every function $f: \Omega \rightarrow R$ let $[f]_{\chi, \mathcal{S}}$ be the set given by $[f]_{\chi, \mathcal{S}}=\{g: \Omega \rightarrow S:[(\exists A \in \mathcal{F}: \chi(A)=0$ and $\{\omega \in \Omega: f(\omega) \neq g(\omega)\} \subseteq$ $\left.\left.A) \cap\left(\forall A \in \mathcal{S}: g^{-1}(A) \in \mathcal{F}\right)\right]\right\}$. Let $T, \nu \in(0, \infty),\left(H,\langle\cdot, \cdot\rangle_{H},\|\cdot\|_{H}\right)=\left(L^{2}\left(\lambda_{(0,1)} ; \mathbb{R}\right)\right.$, $\left.\langle\cdot, \cdot\rangle_{L^{2}\left(\lambda_{(0,1)} ; \mathbb{R}\right)},\|\cdot\|_{L^{2}\left(\lambda_{(0,1)} ; \mathbb{R}\right)}\right),\left(e_{n}\right)_{n \in \mathbb{N}} \subseteq H,\left(P_{n}\right)_{n \in \mathbb{N} \cup\{\infty\}} \subseteq L(H)$ satisfy for all $m \in \mathbb{N}, n \in$ $\mathbb{N} \cup\{\infty\}, u \in H$ that $e_{m}=\left[(\sqrt{2} \sin (m \pi x))_{x \in(0,1)}\right]_{\lambda_{(0,1)}, \mathcal{B}(\mathbb{R})}$ and $P_{n}(u)=\sum_{k=1}^{n}\left\langle e_{k}, u\right\rangle_{H} e_{k}$. Let $A: D(A) \subseteq H \rightarrow H$ be the Laplacian with zero Dirichlet boundary conditions on $H$ times the real number $\nu$. Let $(\Omega, \mathcal{F}, \mathbb{P})$ be a probability space. Let $\left(W_{t}\right)_{t \in[0, T]}$ be an $\operatorname{Id}_{H^{-}}$cylindrical Wiener process. Let $O:[0, T] \times \Omega \rightarrow H$ and $\mathcal{O}^{M, N}:[0, T] \times \Omega \rightarrow H$, $M, N \in \mathbb{N}$, be stochastic processes which satisfy for all $t \in[0, T], M \in \mathbb{N}, N \in \mathbb{N} \cup\{\infty\}$ that $\left[O_{t}\right]_{\mathbb{P}, \mathcal{B}(H)}=\int_{0}^{t} e^{(t-s) A} d W_{s}$ and $\left[\mathcal{O}_{t}^{M, N}\right]_{\mathbb{P}, \mathcal{B}(H)}=\int_{0}^{t} P_{N} e^{\left(t-\lfloor s\rfloor_{T / M}\right) A} d W_{s}$.

\subsection{Lower and upper bounds for Hilbert-Schmidt norms of Hilbert- Schmidt operators}

Lemma 2.1. Assume the setting in Section 2.1 and let $N \in \mathbb{N} \cup\{\infty\}, s_{1}, s_{2}, t \in[0, \infty)$ with $s_{1} \leq s_{2}$. Then

(i) we have that

$$
\left(\sum_{n=1}^{\infty}\left\|P_{N} e^{s_{1} A}\left(\operatorname{Id}_{H}-e^{t A}\right) e_{n}\right\|_{H}^{2}\right)^{1 / 2} \geq\left(\sum_{n=1}^{\infty}\left\|P_{N} e^{s_{2} A}\left(\operatorname{Id}_{H}-e^{t A}\right) e_{n}\right\|_{H}^{2}\right)^{1 / 2}
$$

and

(ii) we have that

$$
\left(\sum_{n=1}^{\infty}\left\|P_{N} e^{t A}\left(\operatorname{Id}_{H}-e^{s_{1} A}\right) e_{n}\right\|_{H}^{2}\right)^{1 / 2} \leq\left(\sum_{n=1}^{\infty}\left\|P_{N} e^{t A}\left(\operatorname{Id}_{H}-e^{s_{2} A}\right) e_{n}\right\|_{H}^{2}\right)^{1 / 2}
$$


Proof of Lemma 2.1. Throughout this proof let $\left(\mu_{n}\right)_{n \in \mathbb{N}} \subseteq \mathbb{R}$ satisfy for all $n \in \mathbb{N}$ that $\mu_{n}=\nu \pi^{2} n^{2}$. Next observe that

$$
\begin{aligned}
& \sum_{n=1}^{\infty}\left\|P_{N} e^{s_{1} A}\left(\operatorname{Id}_{H}-e^{t A}\right) e_{n}\right\|_{H}^{2} \\
& =\sum_{n=1}^{N}\left\|e^{s_{1} A}\left(\operatorname{Id}_{H}-e^{t A}\right) e_{n}\right\|_{H}^{2}=\sum_{n=1}^{N}\left\|e^{-\mu_{n} s_{1}}\left(1-e^{-\mu_{n} t}\right) e_{n}\right\|_{H}^{2} \\
& =\sum_{n=1}^{N}\left|e^{-\mu_{n} s_{1}}\left(1-e^{-\mu_{n} t}\right)\right|^{2} \geq \sum_{n=1}^{N}\left|e^{-\mu_{n} s_{2}}\left(1-e^{-\mu_{n} t}\right)\right|^{2} \\
& =\sum_{n=1}^{N}\left\|e^{s_{2} A}\left(\operatorname{Id}_{H}-e^{t A}\right) e_{n}\right\|_{H}^{2}=\sum_{n=1}^{\infty}\left\|P_{N} e^{s_{2} A}\left(\operatorname{Id}_{H}-e^{t A}\right) e_{n}\right\|_{H}^{2}
\end{aligned}
$$

This establishes (ii). Moreover, note that

$$
\begin{aligned}
& \sum_{n=1}^{\infty}\left\|P_{N} e^{t A}\left(\operatorname{Id}_{H}-e^{s_{1} A}\right) e_{n}\right\|_{H}^{2} \\
& =\sum_{n=1}^{N}\left\|e^{t A}\left(\operatorname{Id}_{H}-e^{s_{1} A}\right) e_{n}\right\|_{H}^{2}=\sum_{n=1}^{N}\left\|e^{-\mu_{n} t}\left(1-e^{-\mu_{n} s_{1}}\right) e_{n}\right\|_{H}^{2} \\
& =\sum_{n=1}^{N}\left|e^{-\mu_{n} t}\left(1-e^{-\mu_{n} s_{1}}\right)\right|^{2} \leq \sum_{n=1}^{N}\left|e^{-\mu_{n} t}\left(1-e^{-\mu_{n} s_{2}}\right)\right|^{2} \\
& =\sum_{n=1}^{N}\left\|e^{t A}\left(\operatorname{Id}_{H}-e^{s_{2} A}\right) e_{n}\right\|_{H}^{2}=\sum_{n=1}^{\infty}\left\|P_{N} e^{t A}\left(\operatorname{Id}_{H}-e^{s_{2} A}\right) e_{n}\right\|_{H}^{2}
\end{aligned}
$$

The proof of Lemma 2.1 is thus completed.

Lemma 2.2. Assume the setting in Section 2.1 and let $N \in \mathbb{N} \cup\{\infty\}, t \in(0, T]$. Then

$$
\begin{gathered}
{\left[\int_{0}^{\max \left\{0, t(N+1)^{2}-(1+\sqrt{t})^{2}\right\}} \frac{\left(1-e^{-\nu \pi^{2} \min \left\{1, t N^{2}\right\}}\right)^{2}}{2 \nu \pi^{2}\left(x+[1+\sqrt{T}]^{2}\right)^{3 / 2}} d x\right]^{1 / 2}} \\
\quad \leq\left\|P_{N}(-\sqrt{t} A)^{-1 / 2}\left(\operatorname{Id}_{H}-e^{t A}\right)\right\|_{H S(H)} \leq\left[\frac{1}{\pi \sqrt{\nu}}+\frac{1}{\nu \pi^{2}}+4 \pi \sqrt{\nu}\right]^{1 / 2}
\end{gathered}
$$


Proof of Lemma 2.2. Observe that

$$
\begin{aligned}
& \frac{1}{\sqrt{t}}\left\|P_{N}(-A)^{-1 / 2}\left(\operatorname{Id}_{H}-e^{t A}\right)\right\|_{H S(H)}^{2} \\
& =\frac{1}{\sqrt{t}} \sum_{k=1}^{N}\left\|(-A)^{-1 / 2}\left(\operatorname{Id}_{H}-e^{t A}\right) e_{k}\right\|_{H}^{2} \\
& =\frac{1}{\sqrt{t}} \sum_{k=1}^{N}\left\|\left(\nu \pi^{2} k^{2}\right)^{-1 / 2}\left(1-e^{-\nu \pi^{2} k^{2} t}\right) e_{k}\right\|_{H}^{2} \\
& =\sum_{k=1}^{N} \frac{\left(1-e^{-\nu \pi^{2} k^{2} t}\right)^{2}}{\nu \pi^{2} k^{2} \sqrt{t}}=\sum_{k=1}^{N} \int_{k}^{k+1} \frac{\left(1-e^{-\nu \pi^{2} k^{2} t}\right)^{2}}{\nu \pi^{2} k^{2} \sqrt{t}} d x \\
& \geq \sum_{k=1}^{N} \int_{k}^{k+1} \frac{\left(1-e^{-\nu \pi^{2}(x-1)^{2} t}\right)^{2}}{\nu \pi^{2} x^{2} \sqrt{t}} d x \\
& =\int_{1}^{N+1} \frac{\left(1-e^{-\nu \pi^{2}(x-1)^{2} t}\right)^{2}}{\nu \pi^{2} x^{2} \sqrt{t}} d x \\
& \geq \int_{1+\min \{1 / \sqrt{t}, N\}}^{N+1} \frac{\left(1-e^{-\nu \pi^{2}(x-1)^{2} t}\right)^{2}}{\nu \pi^{2} x^{2} \sqrt{t}} d x .
\end{aligned}
$$

This and the integral transformation theorem imply that

$$
\begin{aligned}
& \frac{1}{\sqrt{t}}\left\|P_{N}(-A)^{-1 / 2}\left(\operatorname{Id}_{H}-e^{t A}\right)\right\|_{H S(H)}^{2} \\
& \geq \int_{1+\min \{1 / \sqrt{t}, N\}}^{N+1} \frac{\left(1-e^{-\nu \pi^{2} \min \left\{1, t N^{2}\right\}}\right)^{2}}{\nu \pi^{2} x^{2} \sqrt{t}} d x \\
& =\int_{(1+\min \{1 / \sqrt{t}, N\})^{2}}^{(N+1)^{2}} \frac{\left(1-e^{-\nu \pi^{2} \min \left\{1, t N^{2}\right\}}\right)^{2}}{2 \nu \pi^{2} x \sqrt{x t}} d x \\
& =\int_{t(1+\min \{1 / \sqrt{t}, N\})^{2}}^{t(N+1)^{2}} \frac{\left(1-e^{-\nu \pi^{2} \min \left\{1, t N^{2}\right\}}\right)^{2}}{2 \nu \pi^{2} x \sqrt{x}} d x \\
& =\int_{\min \left\{(1+\sqrt{t})^{2}, t(N+1)^{2}\right\}}^{t(N+1)^{2}} \frac{\left(1-e^{-\nu \pi^{2} \min \left\{1, t N^{2}\right\}}\right)^{2}}{2 \nu \pi^{2} x \sqrt{x}} d x \\
& =\int_{0}^{t(N+1)^{2}-\min \left\{(1+\sqrt{t})^{2}, t(N+1)^{2}\right\}} \frac{\left(1-e^{-\nu \pi^{2} \min \left\{1, t N^{2}\right\}}\right)^{2}}{2 \nu \pi^{2}\left(x+\min \left\{(1+\sqrt{t})^{2}, t(N+1)^{2}\right\}\right)^{3 / 2}} d x \\
& \geq \int_{0}^{\max \left\{0, t(N+1)^{2}-(1+\sqrt{t})^{2}\right\}} \frac{\left(1-e^{-\nu \pi^{2} \min \left\{1, t N^{2}\right\}}\right)^{2}}{2 \nu \pi^{2}\left(x+[1+\sqrt{T}]^{2}\right)^{3 / 2}} d x .
\end{aligned}
$$


Moreover, note that

$$
\begin{aligned}
& \frac{1}{\sqrt{t}}\left\|P_{N}(-A)^{-1 / 2}\left(\operatorname{Id}_{H}-e^{t A}\right)\right\|_{H S(H)}^{2} \\
& =\frac{1}{\sqrt{t}} \sum_{k=1}^{N}\left\|(-A)^{-1 / 2}\left(\operatorname{Id}_{H}-e^{t A}\right) e_{k}\right\|_{H}^{2} \\
& =\frac{1}{\sqrt{t}} \sum_{k=1}^{N}\left\|\left(\nu \pi^{2} k^{2}\right)^{-1 / 2}\left(1-e^{-\nu \pi^{2} k^{2} t}\right) e_{k}\right\|_{H}^{2} \\
& =\sum_{k=1}^{N} \frac{\left(1-e^{-\nu \pi^{2} k^{2} t}\right)^{2}}{\nu \pi^{2} k^{2} \sqrt{t}} \\
& =\frac{\left(1-e^{-\nu \pi^{2} t}\right)^{2}}{\nu \pi^{2} \sqrt{t}}+\sum_{k=2}^{N} \int_{k-1}^{k} \frac{\left(1-e^{-\nu \pi^{2} k^{2} t}\right)^{2}}{\nu \pi^{2} k^{2} \sqrt{t}} d x .
\end{aligned}
$$

The fact that

$$
\forall x \in(0, \infty), r \in[0,1]: x^{-r}\left(1-e^{-x}\right) \leq 1,
$$

the fact that

$$
\forall x \in[1, \infty):(x+1)^{2} \leq 4 x^{2},
$$

and the integral transformation theorem hence yield that

$$
\begin{aligned}
& \frac{1}{\sqrt{t}}\left\|P_{N}(-A)^{-1 / 2}\left(\operatorname{Id}_{H}-e^{t A}\right)\right\|_{H S(H)}^{2} \\
& \leq \frac{\left(1-e^{-\nu \pi^{2} t}\right)^{3 / 2}}{\pi \sqrt{\nu}}+\sum_{k=2}^{N} \int_{k-1}^{k} \frac{\left(1-e^{-\nu \pi^{2}(x+1)^{2} t}\right)^{2}}{\nu \pi^{2} x^{2} \sqrt{t}} d x \\
& \leq \frac{1}{\pi \sqrt{\nu}}+\int_{1}^{N} \frac{\left(1-e^{-4 \nu \pi^{2} x^{2} t}\right)^{2}}{\nu \pi^{2} x^{2} \sqrt{t}} d x \\
& =\frac{1}{\pi \sqrt{\nu}}+\int_{1}^{N^{2}} \frac{\left(1-e^{-4 \nu \pi^{2} x t}\right)^{2}}{2 \nu \pi^{2} x \sqrt{x t}} d x \\
& =\frac{1}{\pi \sqrt{\nu}}+\int_{t}^{t N^{2}} \frac{\left(1-e^{-4 \nu \pi^{2} x}\right)^{2}}{2 \nu \pi^{2} x \sqrt{x}} d x .
\end{aligned}
$$

Again the fact that

$$
\forall x \in(0, \infty), r \in[0,1]: x^{-r}\left(1-e^{-x}\right) \leq 1
$$


therefore ensures that

$$
\begin{aligned}
& \frac{1}{\sqrt{t}}\left\|P_{N}(-A)^{-1 / 2}\left(\operatorname{Id}_{H}-e^{t A}\right)\right\|_{H S(H)}^{2} \\
& \leq \frac{1}{\pi \sqrt{\nu}}+\int_{0}^{\infty} \frac{\left(1-e^{-4 \nu \pi^{2} x}\right)^{2}}{2 \nu \pi^{2} x \sqrt{x}} d x \\
& \leq \frac{1}{\pi \sqrt{\nu}}+2 \int_{0}^{1} \frac{\left(1-e^{-4 \nu \pi^{2} x}\right)}{\sqrt{x}} d x+\int_{1}^{\infty} \frac{1}{2 \nu \pi^{2} x \sqrt{x}} d x \\
& \leq \frac{1}{\pi \sqrt{\nu}}+4 \pi \sqrt{\nu} \int_{0}^{1} \sqrt{1-e^{-4 \nu \pi^{2} x}} d x+\left[\frac{-1}{\nu \pi^{2} \sqrt{x}}\right]_{x=1}^{x=\infty} \\
& \leq \frac{1}{\pi \sqrt{\nu}}+4 \pi \sqrt{\nu}+\frac{1}{\nu \pi^{2}} .
\end{aligned}
$$

Combining this and (12) completes the proof of Lemma 2.2.

\subsection{Lower and upper bounds for strong approximation errors of temporal discretizations of linear stochastic heat equations}

Lemma 2.3. Assume the setting in Section [2.1 and let $M \in \mathbb{N}, N \in \mathbb{N} \cup\{\infty\}$. Then

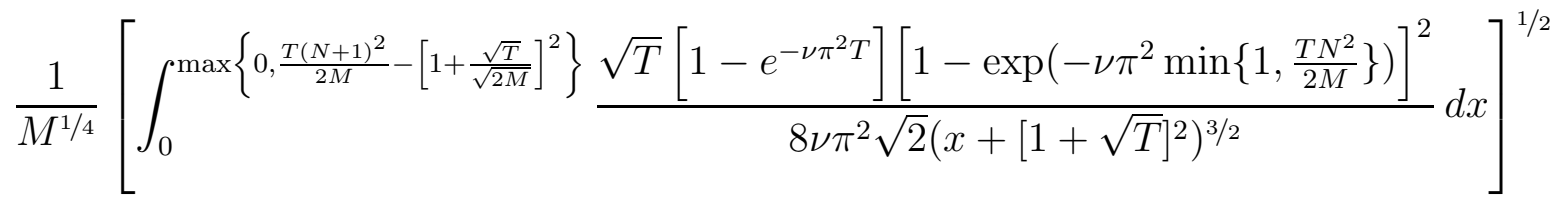

$$
\begin{aligned}
& \leq\left\|P_{N} O_{T}-\mathcal{O}_{T}^{M, N}\right\|_{\mathcal{L}^{2}(\mathbb{P} ; H)} \leq \sup _{t \in[0, T]}\left\|P_{N} O_{t}-\mathcal{O}_{t}^{M, N}\right\|_{\mathcal{L}^{2}(\mathbb{P} ; H)} \\
& =\sup _{t \in[0, T]}\left[\int_{0}^{t}\left\|P_{N} e^{(t-s) A}\left(\operatorname{Id}_{H}-e^{\left(s-\lfloor s\rfloor_{T / M}\right) A}\right)\right\|_{H S(H)}^{2} d s\right]^{1 / 2} \\
& \leq \frac{1}{M^{1 / 4}}\left[\frac{\sqrt{T}}{2}\left(\frac{1}{\pi \sqrt{\nu}}+\frac{1}{\nu \pi^{2}}+4 \pi \sqrt{\nu}\right)\right]^{1 / 2} \text {. }
\end{aligned}
$$

Proof of Lemma 2.3. Throughout this proof let $\left(\mu_{n}\right)_{n \in \mathbb{N}} \subseteq \mathbb{R}$ satisfy for all $n \in \mathbb{N}$ that $\mu_{n}=\nu \pi^{2} n^{2}$ and let $\lceil\cdot\rceil_{h}: \mathbb{R} \rightarrow \mathbb{R}, h \in(0, \infty)$, be the functions which satisfy for all $h \in(0, \infty), t \in \mathbb{R}$ that $\lceil t\rceil_{h}=\min (\{0, h,-h, 2 h,-2 h, \ldots\} \cap[t, \infty))$. Observe that 
Lemma 2.1 (ii) ensures for all $t \in[0, T)$ that

$$
\begin{aligned}
2 & \int_{\lfloor t\rfloor_{T / M}}^{\lfloor t\rfloor_{T / M}+\frac{T}{M}} \mathbb{1}_{\left[\lfloor s\rfloor_{T / M},\lfloor s\rfloor_{T / M}+\frac{T}{2 M}\right]}^{\mathbb{R}}(s)\left\|P_{N} e^{s A}\left(\operatorname{Id}_{H}-e^{\frac{T}{2 M} A}\right)\right\|_{H S(H)}^{2} d s \\
= & 2 \int_{\lfloor t\rfloor_{T / M}}^{\lfloor\rfloor_{T / M}+\frac{T}{2 M}}\left\|P_{N} e^{s A}\left(\operatorname{Id}_{H}-e^{\frac{T}{2 M} A}\right)\right\|_{H S(H)}^{2} d s \\
\geq & \int_{\lfloor t\rfloor_{T / M}}^{\lfloor t\rfloor_{T / M}+\frac{T}{2 M}}\left\|P_{N} e^{s A}\left(\operatorname{Id}_{H}-e^{\frac{T}{2 M} A}\right)\right\|_{H S(H)}^{2} d s \\
& +\int_{\lfloor t\rfloor_{T / M}+\frac{T}{2 M}}^{\lfloor t\rfloor_{T / M}+\frac{T}{M}}\left\|P_{N} e^{s A}\left(\operatorname{Id}_{H}-e^{\frac{T}{2 M} A}\right)\right\|_{H S(H)}^{2} d s \\
= & \int_{\lfloor t\rfloor_{T / M}}^{\lfloor t\rfloor_{T / M}+\frac{T}{M}}\left\|P_{N} e^{s A}\left(\operatorname{Id}_{H}-e^{\frac{T}{2 M} A}\right)\right\|_{H S(H)}^{2} d s .
\end{aligned}
$$

Therefore, we obtain that

$$
\begin{aligned}
& 2 \int_{0}^{T} \mathbb{1}_{\left[[s\rfloor_{T / M},\lfloor s\rfloor_{T / M}+\frac{T}{2 M}\right]}^{\mathbb{R}}(s)\left\|P_{N} e^{s A}\left(\operatorname{Id}_{H}-e^{\frac{T}{2 M} A}\right)\right\|_{H S(H)}^{2} d s \\
& \geq \int_{0}^{T}\left\|P_{N} e^{s A}\left(\operatorname{Id}_{H}-e^{\frac{T}{2 M} A}\right)\right\|_{H S(H)}^{2} d s .
\end{aligned}
$$

Next note that Itô's isometry implies for all $t \in[0, T]$ that

$$
\begin{aligned}
& \left\|P_{N} O_{t}-\mathcal{O}_{t}^{M, N}\right\|_{\mathcal{L}^{2}(\mathbb{P} ; H)}^{2} \\
& =\mathbb{E}\left[\left\|P_{N} O_{t}-\mathcal{O}_{t}^{M, N}\right\|_{H}^{2}\right]=\mathbb{E}\left[\left\|\int_{0}^{t} P_{N} e^{(t-s) A}\left(\operatorname{Id}_{H}-e^{\left(s-\lfloor s\rfloor_{T / M}\right) A}\right) d W_{s}\right\|_{H}^{2}\right] \\
& =\int_{0}^{t}\left\|P_{N} e^{(t-s) A}\left(\operatorname{Id}_{H}-e^{\left(s-\lfloor s\rfloor_{T / M}\right) A}\right)\right\|_{H S(H)}^{2} d s .
\end{aligned}
$$


This, the fact that $\forall s \in[0, T]: T-\lfloor T-s\rfloor_{T / M}=\lceil s\rceil_{T / M}$, and Lemma 2.1 (ii) ensure that

$$
\begin{aligned}
& \left\|P_{N} O_{T}-\mathcal{O}_{T}^{M, N}\right\|_{\mathcal{L}^{2}(\mathbb{P} ; H)}^{2} \\
& =\int_{0}^{T}\left\|P_{N} e^{s A}\left(\operatorname{Id}_{H}-e^{\left(T-s-\lfloor T-s\rfloor_{T / M}\right) A}\right)\right\|_{H S(H)}^{2} d s \\
& =\int_{0}^{T}\left\|P_{N} e^{s A}\left(\operatorname{Id}_{H}-e^{\left([s\rceil_{T / M}-s\right) A}\right)\right\|_{H S(H)}^{2} d s \\
& \geq \int_{0}^{T} \mathbb{1}_{\left[\lfloor s\rfloor_{T / M},\lfloor s\rfloor_{T / M}+\frac{T}{2 M}\right]}^{\mathbb{R}}(s)\left\|P_{N} e^{s A}\left(\operatorname{Id}_{H}-e^{\left(\lceil s\rceil_{T / M}-s\right) A}\right)\right\|_{H S(H)}^{2} d s \\
& \geq \int_{0}^{T} \mathbb{1}_{\left[\lfloor s\rfloor_{T / M},\lfloor s\rfloor_{T / M}+\frac{T}{2 M}\right]}^{\mathbb{R}}(s)\left\|P_{N} e^{s A}\left(\operatorname{Id}_{H}-e^{\frac{T}{2 M} A}\right)\right\|_{H S(H)}^{2} d s .
\end{aligned}
$$

Inequality (21) hence proves that

$$
\begin{aligned}
& \left\|P_{N} O_{T}-\mathcal{O}_{T}^{M, N}\right\|_{\mathcal{L}^{2}(\mathbb{P} ; H)}^{2} \\
& \geq \frac{1}{2}\left[2 \int_{0}^{T} \mathbb{1}_{\left[\lfloor s\rfloor_{T / M},\lfloor s\rfloor_{T / M}+\frac{T}{2 M}\right]}^{\mathbb{R}}(s)\left\|P_{N} e^{s A}\left(\operatorname{Id}_{H}-e^{\frac{T}{2 M} A}\right)\right\|_{H S(H)}^{2} d s\right] \\
& \geq \frac{1}{2} \int_{0}^{T}\left\|P_{N} e^{s A}\left(\operatorname{Id}_{H}-e^{\frac{T}{2 M} A}\right)\right\|_{H S(H)}^{2} d s \\
& =\frac{1}{2} \int_{0}^{T} \sum_{k=1}^{N}\left\|e^{s A}\left(\operatorname{Id}_{H}-e^{\frac{T}{2 M} A}\right) e_{k}\right\|_{H}^{2} d s \\
& =\frac{1}{2} \int_{0}^{T} \sum_{k=1}^{N}\left|e^{-\mu_{k} s}\left(1-e^{-\mu_{k} \frac{T}{2 M}}\right)\right|^{2} d s \\
& =\frac{1}{2} \sum_{k=1}^{N} \frac{\left(1-e^{-2 \mu_{k} T}\right)}{2 \mu_{k}} \mid 1-e^{-\left.\mu_{k} \frac{T}{2 M}\right|^{2} .}
\end{aligned}
$$


Lemma 2.2 therefore implies that

$$
\begin{aligned}
\| & P_{N} O_{T}-\mathcal{O}_{T}^{M, N} \|_{\mathcal{L}^{2}(\mathbb{P} ; H)}^{2} \\
\geq & \frac{1}{4}\left(1-e^{-\mu_{1} T}\right) \sum_{k=1}^{N}\left|\frac{\left(1-e^{-\mu_{k} \frac{T}{2 M}}\right)}{\sqrt{\mu_{k}}}\right|^{2} \\
= & \frac{1}{4}\left(1-e^{-\mu_{1} T}\right) \sum_{k=1}^{N}\left\|(-A)^{-1 / 2}\left(\operatorname{Id}_{H}-e^{\frac{T}{2 M} A}\right) e_{k}\right\|_{H}^{2} \\
= & \frac{1}{4}\left(1-e^{-\mu_{1} T}\right)\left\|P_{N}(-A)^{-1 / 2}\left(\operatorname{Id}_{H}-e^{\frac{T}{2 M} A}\right)\right\|_{H S(H)}^{2} \\
\geq & \frac{\sqrt{T}\left(1-e^{-\mu_{1} T}\right)}{4 \sqrt{2 M}} \\
& \cdot\left[\int_{0}^{\max \left\{0, \frac{T(N+1)^{2}}{2 M}-\left[1+\frac{\sqrt{T}}{\sqrt{2 M}}\right]^{2}\right\}} \frac{\left[1-\exp \left(-\nu \pi^{2} \min \left\{1, \frac{T N^{2}}{2 M}\right\}\right)\right]^{2}}{2 \nu \pi^{2}\left(x+[1+\sqrt{T}]^{2}\right)^{3 / 2}} d x\right] .
\end{aligned}
$$

In the next step observe that (22) and Lemma 2.1 (iii) assure that

$$
\begin{aligned}
\sup _{t \in[0, T]}\left\|P_{N} O_{t}-\mathcal{O}_{t}^{M, N}\right\|_{\mathcal{L}^{2}(\mathbb{P} ; H)}^{2} & \leq \sup _{t \in[0, T]} \int_{0}^{t}\left\|P_{N} e^{(t-s) A}\left(\operatorname{Id}_{H}-e^{\frac{T}{M} A}\right)\right\|_{H S(H)}^{2} d s \\
& =\sup _{t \in[0, T]} \int_{0}^{t}\left\|P_{N} e^{s A}\left(\operatorname{Id}_{H}-e^{\frac{T}{M} A}\right)\right\|_{H S(H)}^{2} d s \\
& =\int_{0}^{T}\left\|P_{N} e^{s A}\left(\operatorname{Id}_{H}-e^{\frac{T}{M} A}\right)\right\|_{H S(H)}^{2} d s \\
& =\int_{0}^{T} \sum_{k=1}^{N}\left\|e^{s A}\left(\operatorname{Id}_{H}-e^{\frac{T}{M} A}\right) e_{k}\right\|_{H}^{2} d s .
\end{aligned}
$$


Lemma 2.2 hence yields that

$$
\begin{aligned}
& \sup _{t \in[0, T]}\left\|P_{N} O_{t}-\mathcal{O}_{t}^{M, N}\right\|_{\mathcal{L}^{2}(\mathbb{P} ; H)}^{2} \\
& \leq \int_{0}^{T} \sum_{k=1}^{N}\left|e^{-\mu_{k} s}\left(1-e^{-\mu_{k} \frac{T}{M}}\right)\right|^{2} d s=\sum_{k=1}^{N} \frac{\left(1-e^{-2 \mu_{k} T}\right)}{2 \mu_{k}}\left|1-e^{-\mu_{k} \frac{T}{M}}\right|^{2} \\
& \leq \frac{1}{2} \sum_{k=1}^{N}\left|\frac{\left(1-e^{-\mu_{k} \frac{T}{M}}\right)}{\sqrt{\mu_{k}}}\right|^{2}=\frac{1}{2} \sum_{k=1}^{N}\left\|(-A)^{-1 / 2}\left(\operatorname{Id}_{H}-e^{\frac{T}{M} A}\right) e_{k}\right\|_{H}^{2} \\
& =\frac{1}{2}\left\|P_{N}(-A)^{-1 / 2}\left(\operatorname{Id}_{H}-e^{\frac{T}{M} A}\right)\right\|_{H S(H)}^{2} \leq \frac{\sqrt{T}}{2 \sqrt{M}}\left[\frac{1}{\pi \sqrt{\nu}}+\frac{1}{\nu \pi^{2}}+4 \pi \sqrt{\nu}\right] .
\end{aligned}
$$

Combining this with (22) and (25) completes the proof of Lemma 2.3.

In the next result, Corollary 2.4, we specialize Lemma 2.3 to the case $N=\infty$ where no spatial discretization is applied to the stochastic process $O:[0, T] \times \Omega \rightarrow H$.

Corollary 2.4. Assume the setting in Section 2.1 and let $M \in \mathbb{N}$. Then

$$
\begin{aligned}
& \frac{1}{M^{1 / 4}}\left[\int_{0}^{\infty} \frac{\sqrt{T}\left(1-e^{-\nu \pi^{2} T}\right)\left(1-e^{-\nu \pi^{2}}\right)^{2}}{8 \nu \pi^{2} \sqrt{2}\left(x+[1+\sqrt{T}]^{2}\right)^{3 / 2}} d x\right]^{1 / 2} \\
& \leq \liminf _{N \rightarrow \infty}\left\|P_{N} O_{T}-\mathcal{O}_{T}^{M, N}\right\|_{\mathcal{L}^{2}(\mathbb{P} ; H)}=\limsup _{N \rightarrow \infty}\left\|P_{N} O_{T}-\mathcal{O}_{T}^{M, N}\right\|_{\mathcal{L}^{2}(\mathbb{P} ; H)} \\
& =\left\|O_{T}-\mathcal{O}_{T}^{M, \infty}\right\|_{\mathcal{L}^{2}(\mathbb{P} ; H)} \leq \liminf _{N \rightarrow \infty} \sup _{t \in[0, T]}\left\|P_{N} O_{t}-\mathcal{O}_{t}^{M, N}\right\|_{\mathcal{L}^{2}(\mathbb{P} ; H)} \\
& =\limsup _{N \rightarrow \infty} \sup _{t \in[0, T]}\left\|P_{N} O_{t}-\mathcal{O}_{t}^{M, N}\right\|_{\mathcal{L}^{2}(\mathbb{P} ; H)}=\sup _{t \in[0, T]}\left\|O_{t}-\mathcal{O}_{t}^{M, \infty}\right\|_{\mathcal{L}^{2}(\mathbb{P} ; H)} \\
& \leq \frac{1}{M^{1 / 4}}\left[\frac{\sqrt{T}}{2}\left(\frac{1}{\pi \sqrt{\nu}}+\frac{1}{\nu \pi^{2}}+4 \pi \sqrt{\nu}\right)\right]^{1 / 2} .
\end{aligned}
$$

\subsection{Lower and upper bounds for strong approximation errors of spatial discretizations of linear stochastic heat equations}

Lemma 2.5. Assume the setting in Section 2.1. Then

$$
\limsup _{M \rightarrow \infty} \sup _{N \in \mathbb{N}} \sup _{t \in[0, T]}\left\|\int_{0}^{t}\left(P_{N} e^{(t-s) A}-P_{N} e^{\left(t-\lfloor s\rfloor_{T / M}\right) A}\right) d W_{s}\right\|_{L^{2}(\mathbb{P} ; H)}=0 .
$$


Proof of Lemma 2.5. Throughout this proof let $\alpha \in(0,1 / 4)$ and let $\beta \in(1 / 4,1 / 2-\alpha)$. Note that the fact that $4 \beta>1$ shows that

$$
\begin{aligned}
& \sup _{N \in \mathbb{N}}\left\|P_{N}\right\|_{H S\left(H, H_{-\beta}\right)}^{2} \\
& =\sup _{N \in \mathbb{N}}\left[\sum_{k=1}^{N}\left\|e_{k}\right\|_{H_{-\beta}}^{2}\right]=\sum_{k=1}^{\infty}\left\|(-A)^{-\beta} e_{k}\right\|_{H}^{2} \\
& =\sum_{k=1}^{\infty}\left|\left(\nu \pi^{2} k^{2}\right)^{-\beta}\right|^{2}=\sum_{k=1}^{\infty} \frac{1}{(\sqrt{\nu} \pi k)^{4 \beta}}<\infty .
\end{aligned}
$$

Next observe that for all $M, N \in \mathbb{N}, t \in[0, T]$ we have that

$$
\begin{aligned}
& \int_{0}^{t}\left\|P_{N} e^{(t-s) A}\left(\operatorname{Id}_{H}-e^{\left(s-\lfloor s\rfloor_{T / M}\right) A}\right)\right\|_{H S(H)}^{2} d s \\
& \leq\left\|(-A)^{-\beta} P_{N}\right\|_{H S(H)}^{2} \int_{0}^{t}\left\|(-A)^{\beta} e^{(t-s) A}\left(\operatorname{Id}_{H}-e^{\left(s-\lfloor s\rfloor_{T / M}\right) A}\right)\right\|_{L(H)}^{2} d s \\
& =\left\|P_{N}\right\|_{H S\left(H, H_{-\beta}\right)}^{2} \int_{0}^{t}\left\|(-A)^{(\alpha+\beta)} e^{(t-s) A}(-A)^{-\alpha}\left(\operatorname{Id}_{H}-e^{\left(s-\lfloor s\rfloor_{T / M}\right) A}\right)\right\|_{L(H)}^{2} d s \\
& \leq\left\|P_{N}\right\|_{H S\left(H, H_{-\beta}\right)}^{2} \\
& \quad \cdot \int_{0}^{t}\left\|(-A)^{(\alpha+\beta)} e^{(t-s) A}\right\|_{L(H)}^{2}\left\|(-A)^{-\alpha}\left(\operatorname{Id}_{H}-e^{\left(s-\lfloor s\rfloor_{T / M}\right) A}\right)\right\|_{L(H)}^{2} d s .
\end{aligned}
$$

The fact that

$$
\forall s \in[0, \infty), r \in[0,1]:\left\|(-s A)^{r} e^{s A}\right\|_{L(H)} \leq 1
$$

and the fact that

$$
\forall s \in(0, \infty), r \in[0,1]:\left\|(-s A)^{-r}\left(\operatorname{Id}_{H}-e^{s A}\right)\right\|_{L(H)} \leq 1
$$

hence prove for all $M, N \in \mathbb{N}, t \in[0, T]$ that

$$
\begin{aligned}
& \int_{0}^{t}\left\|P_{N} e^{(t-s) A}\left(\operatorname{Id}_{H}-e^{\left(s-\lfloor s\rfloor_{T / M}\right) A}\right)\right\|_{H S(H)}^{2} d s \\
& \leq\left\|P_{N}\right\|_{H S\left(H, H_{-\beta}\right)}^{2} \int_{0}^{t}(t-s)^{-2(\alpha+\beta)}\left(s-\lfloor s\rfloor_{T / M}\right)^{2 \alpha} d s \\
& \leq \frac{T^{2 \alpha}}{M^{2 \alpha}}\left\|P_{N}\right\|_{H S\left(H, H_{-\beta}\right)}^{2} \int_{0}^{t}(t-s)^{-2(\alpha+\beta)} d s \\
& =\frac{t^{(1-2 \alpha-2 \beta)} T^{2 \alpha}}{(1-2 \alpha-2 \beta) M^{2 \alpha}}\left\|P_{N}\right\|_{H S\left(H, H_{-\beta}\right)}^{2} .
\end{aligned}
$$


Itô's isometry therefore ensures for all $M \in \mathbb{N}$ that

$$
\begin{aligned}
& \sup _{N \in \mathbb{N}} \sup _{t \in[0, T]}\left\|\int_{0}^{t}\left(P_{N} e^{(t-s) A}-P_{N} e^{\left(t-\lfloor s\rfloor_{T / M}\right) A}\right) d W_{s}\right\|_{L^{2}(\mathbb{P} ; H)}^{2} \\
& =\sup _{N \in \mathbb{N}} \sup _{t \in[0, T]} \int_{0}^{t}\left\|P_{N} e^{(t-s) A}-P_{N} e^{\left(t-\lfloor s\rfloor_{T / M}\right) A}\right\|_{H S(H)}^{2} d s \\
& \leq \frac{T^{(1-2 \beta)}}{(1-2 \alpha-2 \beta) M^{2 \alpha}}\left[\sup _{N \in \mathbb{N}}\left\|P_{N}\right\|_{H S\left(H, H_{-\beta}\right)}^{2}\right] .
\end{aligned}
$$

Combining this with (30) completes the proof of Lemma 2.5.

Lemma 2.6. Assume the setting in Section 2.1 and let $N \in \mathbb{N}$. Then

$$
\begin{aligned}
& {\left[\frac{\sqrt{1-e^{-2 \nu \pi^{2} T}}}{2 \pi \sqrt{\nu}}\right] \frac{1}{\sqrt{N}} \leq \liminf _{M \rightarrow \infty}\left\|O_{T}-\mathcal{O}_{T}^{M, N}\right\|_{\mathcal{L}^{2}(\mathbb{P} ; H)}} \\
& =\limsup _{M \rightarrow \infty}\left\|O_{T}-\mathcal{O}_{T}^{M, N}\right\|_{\mathcal{L}^{2}(\mathbb{P} ; H)}=\liminf _{M \rightarrow \infty} \sup _{t \in[0, T]}\left\|O_{t}-\mathcal{O}_{t}^{M, N}\right\|_{\mathcal{L}^{2}(\mathbb{P} ; H)} \\
& =\limsup _{M \rightarrow \infty} \sup _{t \in[0, T]}\left\|O_{t}-\mathcal{O}_{t}^{M, N}\right\|_{\mathcal{L}^{2}(\mathbb{P} ; H)}=\left\|O_{T}-P_{N} O_{T}\right\|_{\mathcal{L}^{2}(\mathbb{P} ; H)} \\
& =\sup _{t \in[0, T]}\left\|O_{t}-P_{N} O_{t}\right\|_{\mathcal{L}^{2}(\mathbb{P} ; H)} \leq\left[\frac{1}{\pi \sqrt{2 \nu}}\right] \frac{1}{\sqrt{N}} .
\end{aligned}
$$

Proof of Lemma 2.6. Throughout this proof let $\left(\mu_{n}\right)_{n \in \mathbb{N}} \subseteq \mathbb{R}$ satisfy for all $n \in \mathbb{N}$ that

$$
\mu_{n}=\nu \pi^{2} n^{2}
$$

Note that Parseval's identity shows that for all $t \in[0, T]$ we have that

$$
\begin{aligned}
& \left\|O_{t}-P_{N} O_{t}\right\|_{\mathcal{L}^{2}(\mathbb{P} ; H)}^{2} \\
& =\mathbb{E}\left[\left\|O_{t}-P_{N} O_{t}\right\|_{H}^{2}\right]=\mathbb{E}\left[\sum_{k=N+1}^{\infty}\left|\left\langle e_{k}, O_{t}\right\rangle_{H}\right|^{2}\right]=\sum_{k=N+1}^{\infty} \mathbb{E}\left[\left|\left\langle e_{k}, O_{t}\right\rangle_{H}\right|^{2}\right] \\
& =\sum_{k=N+1}^{\infty} \mathbb{E}\left[\left|\int_{0}^{t}\left\langle e_{k}, e^{(t-s) A} d W_{s}\right\rangle_{H}\right|^{2}\right]=\sum_{k=N+1}^{\infty} \mathbb{E}\left[\left|\int_{0}^{t}\left\langle e^{(t-s) A} e_{k}, d W_{s}\right\rangle_{H}\right|^{2}\right] .
\end{aligned}
$$


Itô's isometry hence proves for all $t \in[0, T]$ that

$$
\begin{aligned}
& \left\|O_{t}-P_{N} O_{t}\right\|_{\mathcal{L}^{2}(\mathbb{P} ; H)}^{2} \\
& =\sum_{k=N+1}^{\infty} \mathbb{E}\left[\left|\int_{0}^{t} e^{-\mu_{k}(t-s)}\left\langle e_{k}, d W_{s}\right\rangle_{H}\right|^{2}\right] \\
& =\sum_{k=N+1}^{\infty} \int_{0}^{t} e^{-2 \mu_{k}(t-s)} d s=\sum_{k=N+1}^{\infty} \int_{0}^{t} e^{-2 \mu_{k} s} d s=\sum_{k=N+1}^{\infty} \frac{\left(1-e^{-2 \mu_{k} t}\right)}{2 \mu_{k}} .
\end{aligned}
$$

This shows that

$$
\begin{aligned}
& \sup _{t \in[0, T]}\left\|O_{t}-P_{N} O_{t}\right\|_{\mathcal{L}^{2}(\mathbb{P} ; H)}^{2}=\left\|O_{T}-P_{N} O_{T}\right\|_{\mathcal{L}^{2}(\mathbb{P} ; H)}^{2} \\
& =\sum_{k=N+1}^{\infty} \frac{\left(1-e^{-2 \mu_{k} T}\right)}{2 \mu_{k}}=\sum_{k=N+1}^{\infty} \frac{\left(1-e^{-2 \nu \pi^{2} k^{2} T}\right)}{2 \nu \pi^{2} k^{2}} \\
& \geq\left[\frac{1-e^{-2 \nu \pi^{2} T}}{2 \nu \pi^{2}}\right]\left[\sum_{k=N+1}^{\infty} \frac{1}{k^{2}}\right] \geq\left[\frac{1-e^{-2 \nu \pi^{2} T}}{2 \nu \pi^{2}}\right]\left[\sum_{k=N+1}^{\infty} \int_{k}^{k+1} \frac{1}{x^{2}} d x\right] \\
& \left.\left.\left.=\left[\frac{1-e^{-2 \nu \pi^{2} T}}{2 \nu \pi^{2}}\right]\left[\int_{N+1}^{\infty} \frac{1}{x^{2}} d x\right]=\left[\frac{1-e^{-2 \nu \pi^{2} T}}{2 \nu \pi^{2}}\right]\left[-\frac{1}{x}\right]_{x=N+1}^{x=\infty}\right] \frac{1}{2 \nu}\right] \frac{1}{(N+1)} \geq\left[\frac{1-e^{-2 \nu \pi^{2} T}}{2 \nu \pi^{2}}\right] \frac{1-e^{-2 \nu \pi^{2} T}}{4 \nu \pi^{2}}\right] \frac{1}{N} .
\end{aligned}
$$

This implies that

$$
\begin{aligned}
& \sup _{t \in[0, T]}\left\|O_{t}-P_{N} O_{t}\right\|_{\mathcal{L}^{2}(\mathbb{P} ; H)}^{2} \\
& =\sum_{k=N+1}^{\infty} \frac{\left(1-e^{-2 \nu \pi^{2} k^{2} T}\right)}{2 \nu \pi^{2} k^{2}} \leq\left[\frac{1}{2 \nu \pi^{2}}\right]\left[\sum_{k=N+1}^{\infty} \frac{1}{k^{2}}\right] \\
& \leq\left[\frac{1}{2 \nu \pi^{2}}\right]\left[\sum_{k=N+1}^{\infty} \int_{k-1}^{k} \frac{1}{x^{2}} d x\right]=\left[\frac{1}{2 \nu \pi^{2}}\right]\left[\int_{N}^{\infty} \frac{1}{x^{2}} d x\right] \\
& =\left[\frac{1}{2 \nu \pi^{2}}\right]\left[-\frac{1}{x}\right]_{x=N}^{x=\infty}=\left[\frac{1}{2 \nu \pi^{2}}\right] \frac{1}{N} .
\end{aligned}
$$


In addition, note that the triangle inequality and Lemma 2.5 prove that

$$
\begin{aligned}
& \limsup _{M \rightarrow \infty} \sup _{t \in[0, T]}\left\|O_{t}-\mathcal{O}_{t}^{M, N}\right\|_{\mathcal{L}^{2}(\mathbb{P} ; H)} \\
& =\limsup _{M \rightarrow \infty} \sup _{t \in[0, T]}\left\|\int_{0}^{t}\left(e^{(t-s) A}-P_{N} e^{\left(t-\lfloor s\rfloor_{T / M}\right) A}\right) d W_{s}\right\|_{L^{2}(\mathbb{P} ; H)} \\
& =\limsup _{M \rightarrow \infty} \sup _{t \in[0, T]} \| \int_{0}^{t}\left(e^{(t-s) A}-P_{N} e^{(t-s) A}\right) d W_{s} \\
& +\int_{0}^{t}\left(P_{N} e^{(t-s) A}-P_{N} e^{\left(t-\lfloor s\rfloor_{T / M}\right) A}\right) d W_{s} \|_{L^{2}(\mathbb{P} ; H)} \\
& \leq \limsup _{M \rightarrow \infty} \sup _{t \in[0, T]}\left\|\int_{0}^{t}\left(e^{(t-s) A}-P_{N} e^{(t-s) A}\right) d W_{s}\right\|_{L^{2}(\mathbb{P} ; H)} \\
& +\limsup _{M \rightarrow \infty} \sup _{t \in[0, T]}\left\|\int_{0}^{t}\left(P_{N} e^{(t-s) A}-P_{N} e^{\left(t-\lfloor s\rfloor_{T / M}\right) A}\right) d W_{s}\right\|_{L^{2}(\mathbb{P} ; H)} \\
& =\sup _{t \in[0, T]}\left\|\int_{0}^{t}\left(e^{(t-s) A}-P_{N} e^{(t-s) A}\right) d W_{s}\right\|_{L^{2}(\mathbb{P} ; H)}=\sup _{t \in[0, T]}\left\|O_{t}-P_{N} O_{t}\right\|_{\mathcal{L}^{2}(\mathbb{P} ; H)} .
\end{aligned}
$$

Furthermore, observe that the triangle inequality, Lemma 2.5, and (40) ensure that

$$
\begin{aligned}
\liminf _{M \rightarrow \infty}\left\|O_{T}-\mathcal{O}_{T}^{M, N}\right\|_{\mathcal{L}^{2}(\mathbb{P} ; H)} & =\liminf _{M \rightarrow \infty}\left\|\int_{0}^{T}\left(e^{(T-s) A}-P_{N} e^{\left(T-\lfloor s\rfloor_{T / M}\right) A}\right) d W_{s}\right\|_{L^{2}(\mathbb{P} ; H)} \\
= & \liminf _{M \rightarrow \infty} \| \int_{0}^{T}\left(e^{(T-s) A}-P_{N} e^{(T-s) A}\right) d W_{s} \\
& +\int_{0}^{T}\left(P_{N} e^{(T-s) A}-P_{N} e^{\left(T-\lfloor s\rfloor_{T / M}\right) A}\right) d W_{s} \|_{L^{2}(\mathbb{P} ; H)} \\
\geq & \liminf _{M \rightarrow \infty}\left\|\int_{0}^{T}\left(e^{(T-s) A}-P_{N} e^{(T-s) A}\right) d W_{s}\right\|_{L^{2}(\mathbb{P} ; H)} \\
& -\liminf _{M \rightarrow \infty}\left\|\int_{0}^{T}\left(P_{N} e^{(T-s) A}-P_{N} e^{\left(T-\lfloor s\rfloor_{T / M}\right) A}\right) d W_{s}\right\|_{L^{2}(\mathbb{P} ; H)} \\
= & \left\|\int_{0}^{T}\left(e^{(T-s) A}-P_{N} e^{(T-s) A}\right) d W_{s}\right\|_{L^{2}(\mathbb{P} ; H)}=\left\|O_{T}-P_{N} O_{T}\right\|_{\mathcal{L}^{2}(\mathbb{P} ; H)} \\
= & \sup _{t \in[0, T]}\left\|O_{t}-P_{N} O_{t}\right\|_{\mathcal{L}^{2}(\mathbb{P} ; H)}
\end{aligned}
$$


Combining this with (40)-(42) completes the proof of Lemma 2.6.

\subsection{Lower and upper bounds for strong approximation errors of full discretizations of linear stochastic heat equations}

Theorem 2.7. Assume the setting in Section 2.1 and let $M, N \in \mathbb{N}$. Then

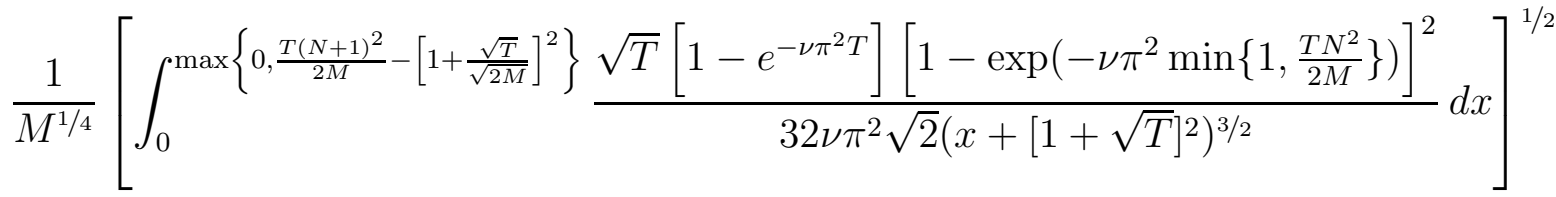

$$
\begin{aligned}
& +\frac{1}{N^{1 / 2}}\left[\frac{\sqrt{1-e^{-\nu T}}}{4 \pi \sqrt{\nu}}\right] \\
& \leq\left\|O_{T}-\mathcal{O}_{T}^{M, N}\right\|_{\mathcal{L}^{2}(\mathbb{P} ; H)} \leq \sup _{t \in[0, T]}\left\|O_{t}-\mathcal{O}_{t}^{M, N}\right\|_{\mathcal{L}^{2}(\mathbb{P} ; H)} \\
& \leq \frac{1}{M^{1 / 4}}\left[\frac{\sqrt{T}}{2}\left(\frac{1}{\pi \sqrt{\nu}}+\frac{1}{\nu \pi^{2}}+4 \pi \sqrt{\nu}\right)\right]^{1 / 2}+\frac{1}{N^{1 / 2}}\left[\frac{1}{\pi \sqrt{2 \nu}}\right]
\end{aligned}
$$

Proof of Theorem 2.7. Observe that the fact that $P_{N}$ is self-adjoint ensures for all $x \in H$, $y \in P_{N}(H)$ that

$$
\begin{aligned}
& \left\langle x-P_{N}(x), P_{N}(x)-y\right\rangle_{H} \\
& =\left\langle x-P_{N}(x), P_{N}(x)-P_{N}(y)\right\rangle_{H}=\left\langle x-P_{N}(x), P_{N}(x-y)\right\rangle_{H} \\
& =\left\langle P_{N}\left(x-P_{N}(x)\right), x-y\right\rangle_{H}=\left\langle P_{N}(x)-P_{N}(x), x-y\right\rangle_{H} \\
& =\langle 0, x-y\rangle_{H}=0 .
\end{aligned}
$$

This implies for all $t \in[0, T]$ that

$$
\begin{aligned}
\left\|O_{t}-\mathcal{O}_{t}^{M, N}\right\|_{\mathcal{L}^{2}(\mathbb{P} ; H)}^{2} & \\
= & \mathbb{E}\left[\left\|O_{t}-\mathcal{O}_{t}^{M, N}\right\|_{H}^{2}\right]=\mathbb{E}\left[\left\|O_{t}-P_{N} O_{t}+P_{N} O_{t}-\mathcal{O}_{t}^{M, N}\right\|_{H}^{2}\right] \\
= & \mathbb{E}\left[\left\|O_{t}-P_{N} O_{t}\right\|_{H}^{2}\right]+2 \mathbb{E}\left[\left\langle O_{t}-P_{N} O_{t}, P_{N} O_{t}-\mathcal{O}_{t}^{M, N}\right\rangle_{H}\right] \\
& +\mathbb{E}\left[\left\|P_{N} O_{t}-\mathcal{O}_{t}^{M, N}\right\|_{H}^{2}\right] \\
= & \left\|O_{t}-P_{N} O_{t}\right\|_{\mathcal{L}^{2}(\mathbb{P} ; H)}^{2}+\left\|P_{N} O_{t}-\mathcal{O}_{t}^{M, N}\right\|_{\mathcal{L}^{2}(\mathbb{P} ; H)}^{2} .
\end{aligned}
$$


Combining this with Lemma 2.3, Lemma 2.6, and the fact that

$$
\forall x, y \in[0, \infty): \sqrt{x} / 2+\sqrt{y} / 2 \leq \max \{\sqrt{x}, \sqrt{y}\} \leq \sqrt{x+y} \leq \sqrt{x}+\sqrt{y}
$$

completes the proof of Theorem 2.7 .

\section{References}

[1] Becker, S., Gess, B., Jentzen, A., Kloeden, P.E.: Strong convergence rates for explicit space-time discrete numerical approximations of stochastic Allen-Cahn equations. arXiv:1711.02423v1 p. 104 pages (2017)

[2] Becker, S., Jentzen, A.: Strong convergence rates for nonlinearity-truncated Eulertype approximations of stochastic Ginzburg-Landau equations. Stoch. Proc. Appl. 129(1), 28-69 (2019)

[3] Bréhier, C.E., Cui, J., Hong, J.: Strong convergence rates of semi-discrete splitting approximations for stochastic Allen-Cahn equation. IMA J. Numer. Anal. 39(4), 2096-2134 (2018)

[4] Bréhier, C.E., Goudenège, L.: Analysis of Some Splitting Schemes for the stochastic Allen-Cahn equation. Discrete Contin. Dyn. Syst. Ser. B 24(8), 4169-4190 (2019)

[5] Conus, D., Jentzen, A., Kurniawan, R.: Weak convergence rates of spectral Galerkin approximations for SPDEs with nonlinear diffusion coefficients. Ann. Appl. Probab. 29(2), 653-716 (2019)

[6] Da Prato, G., Zabczyk, J.: Stochastic equations in infinite dimensions, Encyclopedia of Mathematics and its Applications, vol. 44. Cambridge University Press, Cambridge (1992)

[7] Gyöngy, I., Sabanis, S., Šiška, D.: Convergence of tamed Euler schemes for a class of stochastic evolution equations. Stoch. Partial Differ. Equ. Anal. Comput. 4(2), $225-245(2016)$

[8] Hutzenthaler, M., Jentzen, A.: Numerical approximations of stochastic differential equations with non-globally Lipschitz continuous coefficients. Mem. Amer. Math. Soc. 236(1112), v+99 (2015)

[9] Hutzenthaler, M., Jentzen, A., Kloeden, P.E.: Strong convergence of an explicit numerical method for SDEs with non-globally Lipschitz continuous coefficients. Ann. Appl. Probab. 22(4), 1611-1641 (2012) 
[10] Hutzenthaler, M., Jentzen, A., Kloeden, P.E.: Divergence of the multilevel Monte Carlo Euler method for nonlinear stochastic differential equations. Ann. Appl. Probab. 23(5), 1913-1966 (2013)

[11] Hutzenthaler, M., Jentzen, A., Salimova, D.: Strong convergence of fulldiscrete nonlinearity-truncated accelerated exponential Euler-type approximations for stochastic Kuramoto-Sivashinsky equations. Commun. Math. Sci. 16(6), 14891529 (2018)

[12] Jentzen, A., Kurniawan, R.: Weak convergence rates for Euler-type approximations of semilinear stochastic evolution equations with nonlinear diffusion coefficients. arXiv:1501.03539 p. 51 pages. To appear in Found. Comput. Math.. (2015)

[13] Jentzen, A., Pušnik, P.: Strong convergence rates for an explicit numerical approximation method for stochastic evolution equations with non-globally Lipschitz continuous nonlinearities. IMA J. Numer. Anal., Published online at https://doi.org/10.1093/imanum/drz009 (2019)

[14] Jentzen, A., Pušnik, P.: Exponential moments for numerical approximations of stochastic partial differential equations. Stoch. PDE: Anal. Comp. 6, 565-617 (2018)

[15] Jentzen, A., Salimova, D., Welti, T.: Strong convergence for explicit space-time discrete numerical approximation methods for stochastic Burgers equations. J. Math. Anal. Appl. 469(2), 661-704 (2019)

[16] Müller-Gronbach, T., Ritter, K.: Lower bounds and nonuniform time discretization for approximation of stochastic heat equations. Found. Comput. Math. 7(2), 135-181 (2007)

[17] Müller-Gronbach, T., Ritter, K., Wagner, T.: Optimal pointwise approximation of a linear stochastic heat equation with additive space-time white noise. In: Monte Carlo and quasi-Monte Carlo methods 2006, pp. 577-589. Springer, Berlin (2008)

[18] Müller-Gronbach, T., Ritter, K., Wagner, T.: Optimal pointwise approximation of infinite-dimensional Ornstein-Uhlenbeck processes. Stoch. Dyn. 8(3), 519-541 (2008)

[19] Qi, R., Wang, X.: Optimal error estimates of Galerkin finite element methods for stochastic Allen-Cahn equation with additive noise. arXiv:1804.11331 p. 24 pages (2018)

[20] Sabanis, S.: A note on tamed Euler approximations. Electron. Commun. Probab. 18(47), 1-10 (2013) 
[21] Sabanis, S.: Euler approximations with varying coefficients: the case of superlinearly growing diffusion coefficients. Ann. Appl. Probab. 26(4), 2083-2105 (2016)

[22] Sabanis, S., Zhang, Y.: On explicit order 1.5 approximations with varying coefficients: the case of super-linear diffusion coefficients. J. Complex. 50, 84-115 (2019)

[23] Tretyakov, M.V., Zhang, Z.: A fundamental mean-square convergence theorem for SDEs with locally Lipschitz coefficients and its applications. SIAM J. Numer. Anal. 51(6), 3135-3162 (2013)

[24] Wang, X.: An efficient explicit full discrete scheme for strong approximation of stochastic Allen-Cahn equation. arXiv:1802.09413 p. 25 pages (2018)

[25] Wang, X., Gan, S.: The tamed Milstein method for commutative stochastic differential equations with non-globally Lipschitz continuous coefficients. J. Difference Equ. Appl. 19(3), 466-490 (2013) 